\title{
On polynomial solutions of Heun equation
}

\author{
N. Gurappa* and Prasanta K. Panigrahi ${ }^{\dagger}$ \\ Physical Research Laboratory, Navrangpura, Ahmedabad-380 009, India
}

\begin{abstract}
By making use of a recently developed method to solve linear differential equations of arbitrary order, we find a wide class of polynomial solutions to the Heun equation. We construct the series solution to the Heun equation before identifying the polynomial solutions. The Heun equation extended by the addition of a term, $-\sigma / x$, is also amenable for polynomial solutions.
\end{abstract}

Heun equation, a second order linear differential equation having four regular singularities, is well-known in mathematical literature $[1,2]$ and has appeared in a number of physical problems, like quasi-exactly solvable systems [3], higher dimensional correlated systems [4], Kerr-de Sitter black holes [5], Calogero-Moser-Sutherland systems [6], finite lattice Bethe ansatz systems [7] etc. We analyze this equation by using a newly developed method to solve linear differential equations [8]. This approach to Heun equation is very transparent in obtaining solutions; in particular the polynomial solutions come out very naturally as is shown below. form

A single variable differential equation, after appropriate manipulation, can be cast in the

$$
(F(D)+P(x, d / d x)) y(x)=0 \quad,
$$

where, $D \equiv x \frac{d}{d x}$, and $F(D)=\sum_{n} a_{n} D^{n}$ is a diagonal operator in the space of monomials, with $a_{n}$ 's being some parameters. $P(x, d / d x)$ can be an arbitrary polynomial function of $x$ and $d / d x$, excluding the diagonal operators. It can be shown that the following ansatz,

$$
y(x)=\sum_{m=0}^{\infty}(-1)^{m}\left[\frac{1}{F(D)} P(x, d / d x)\right]^{m} x^{\lambda}
$$

is a solution of the above equation, provided, $F(D) x^{\lambda}=0$ and the coefficient of $x^{\lambda}$ in $y(x)-x^{\lambda}$ is zero, in order to ensure that the solution, $y(x)$, is non-singular [8]. The fact that $D$ is diagonal in the space of monomials, $x^{n}$, makes $1 / F(D)$ well defined in the above expression. For explicating the working of the present method, consider the Jacobi differential equation $[9]$

$$
\left[\left(1-x^{2}\right) \frac{d^{2}}{d x^{2}}+[\beta-\alpha-(\alpha+\beta+2) x] \frac{d}{d x}+n(n+\alpha+\beta+1)\right] P_{n}^{(\alpha, \beta)}(x)=0 \quad,
$$

where, $P_{n}^{(\alpha, \beta)}(x)$ 's are the Jacobi polynomials. Rewriting the above equation in the form of Eq. (1), one finds $F(D)=D^{2}+(\alpha+\beta+1) D-n(n+\alpha+\beta+1)$ and $P(x, d / d x)=$ $-\frac{d^{2}}{d x^{2}}-(\beta-\alpha) \frac{d}{d x} . F(D) x^{\lambda}=0$ yields two solutions $\lambda=n$ and $\lambda=-(n+\alpha+\beta+1)$. From Eq. (2), it is clear that, $\lambda=n$ results in a polynomial solution,

$$
P_{n}^{(\alpha, \beta)}(x)=\sum_{m=0}^{\infty}\left[\frac{1}{(D-n)(D+\alpha+\beta+1)}\left(\frac{d^{2}}{d x^{2}}+(\beta-\alpha) \frac{d}{d x}\right)\right]^{m} x^{n} .
$$

\footnotetext{
* e-mail: nakkagurappa@yahoo.co.in

$\dagger$ e-mail: prasanta@prl.ernet.in
} 
Notice that the choice of $\lambda=-(n+\alpha+\beta+1)$ results in the other linearly independent solution with negative powers of $x$. We now proceed to study the Heun equation by using the above technique. The Heun equation, with four sigularities at $x=0,1, c$ and $\infty$, is given by

$$
\left[\frac{d^{2}}{d x^{2}}+\left(\frac{\gamma}{x}+\frac{\delta}{x-1}+\frac{\epsilon}{x-c}\right) \frac{d}{d x}+\frac{\alpha \beta x-q}{x(x-1)(x-c)}\right] y(x)=0 \quad .
$$

Rewriting the above equation after multiplying it by $x$, one has

$$
\begin{array}{r}
{\left[c x^{2} \frac{d^{2}}{d x^{2}}+\gamma c x \frac{d}{d x}-(1+c) x^{3} \frac{d^{2}}{d x^{2}}-[(1+c) \gamma+\delta c+\epsilon] x^{2} \frac{d}{d x}-q x\right.} \\
\left.+x^{4} \frac{d^{2}}{d x^{2}}+(\gamma+\delta+\epsilon) x^{3} \frac{d}{d x}+\alpha \beta x^{2}\right] y(x)=0
\end{array} .
$$

In the above equation,

$$
c x^{2} \frac{d^{2}}{d x^{2}}+\gamma c x \frac{d}{d x}=F(D)=c D(D+\gamma-1)
$$

and

$$
P(x, d / d x)=A_{+1}+A_{+2} \quad,
$$

where,

$$
A_{+1}=-(1+c) x^{3} \frac{d^{2}}{d x^{2}}-[(1+c) \gamma+\delta c+\epsilon] x^{2} \frac{d}{d x}-q x,
$$

is a degree +1 operator, and

$$
A_{+2}=+x^{4} \frac{d^{2}}{d x^{2}}+(\gamma+\delta+\epsilon) x^{3} \frac{d}{d x}+\alpha \beta x^{2},
$$

is a degree +2 operator, respectively. $d$, the degree of an operator, $O$, is defined from $[D, O]=d O$. Now,

$$
F(D) x^{\lambda}=0,
$$

yields $\lambda=0$ or $1-\gamma$. The solutions, for the values of $\lambda=0$ and $\lambda=1-\gamma$, can be written as

$$
y_{\lambda}(x)=\sum_{m=0}^{\infty}(-c)^{-m}\left[\frac{1}{D(D+\gamma-1)}\left(A_{+1}+A_{+2}\right)\right]^{m} x^{\lambda} .
$$

The above is an infinite series solution to the Heun equation. Below, we look for the polynomial solutions.

Case (i): Rewriting the Heun equation after dividing it by $x$, we get,

$$
\begin{array}{r}
{\left[D^{2}+(\gamma+\delta+\epsilon-1) D+\alpha \beta-(1+c) x \frac{d^{2}}{d x^{2}}-[(1+c) \gamma+\delta c+\epsilon] \frac{d}{d x}-\frac{q}{x}\right.} \\
\left.+c \frac{d^{2}}{d x^{2}}+\gamma c \frac{1}{x} \frac{d}{d x}\right] y(x)=0 .
\end{array}
$$

Here,

$$
F(D)=D^{2}+(\gamma+\delta+\epsilon-1) D+\alpha \beta \quad,
$$


and

$$
\hat{P}=A_{-1}+A_{-2}
$$

where,

and

$$
A_{-1}=-(1+c) x \frac{d^{2}}{d x^{2}}-[(1+c) \gamma+\delta c+\epsilon] \frac{d}{d x}-\frac{q}{x}
$$

$$
A_{-2}=+c \frac{d^{2}}{d x^{2}}+\gamma c \frac{1}{x} \frac{d}{d x}
$$

The condition, $F(D) x^{\lambda}=0$, after imposing the constraint $\gamma+\delta+\epsilon=\alpha+\beta+1$, gives $\lambda=-\alpha$ or $-\beta$. In order to have polynomial solutions, one needs to impose $q=0$ in $A_{-1}$ and furthermore, either $-\alpha$ or $-\beta$ must be a positive integer, say $n$. For the sake of illustration, we choose $-\alpha=n$. Then, the polynomial solutions to the Heun equation, $y_{n}(x)$, can be written as,

$$
y_{n}(x)=\sum_{k=0}^{\infty}(-1)^{n}\left[\frac{1}{(D-n)(D+\beta)}\left(A_{-1}+A_{-2}\right)\right]^{k} x^{n} .
$$

Case(ii): By performing a similarity transformation on Eq.(7) by $x^{(1-\gamma)}$ and writing $y(x)=$ $x^{1-\gamma} \phi_{n}(x)$, the choice of $q=(\delta c+\epsilon)(\gamma-1)$ yields,

$$
\begin{array}{r}
{\left[D^{2}+(1-\gamma+\delta+\epsilon) D+(1-\gamma)(\delta+\epsilon)+\alpha \beta\right.} \\
-(1+c) x \frac{d^{2}}{d x^{2}}-[(1+c)(2-\gamma)+\delta c+\epsilon] \frac{d}{d x} \\
\left.+c \frac{d^{2}}{d x^{2}}+\frac{(2-\gamma) c}{x} \frac{d}{d x}\right] \phi_{n}(x)=0
\end{array}
$$

here,

$$
F(D)=D^{2}+(1-\gamma+\delta+\epsilon) D+(1-\gamma)(\delta+\epsilon)+\alpha \beta
$$

and

$$
P(x, d / d x)=-(1+c) x \frac{d^{2}}{d x^{2}}-[(1+c)(2-\gamma)+\delta c+\epsilon] \frac{d}{d x}+c \frac{d^{2}}{d x^{2}}+\frac{(2-\gamma) c}{x} \frac{d}{d x}
$$

$F(D) x^{\lambda}=0$ results in $\lambda=\gamma-1-\beta$ or $\gamma-1-\alpha$, after imposing the constraint $\gamma+\delta+\epsilon=$ $\alpha+\beta+1$. In order to have polynomial solutions, either $\gamma-1-\beta$ or $\gamma-1-\alpha$ must be a positive integer. Let $\gamma-1-\beta=n$, a positive integer, then the solution is

$$
y_{n}(x)=x^{(1-\gamma)} \sum_{m=0}^{\infty}(-1)^{m}\left[\frac{1}{(D-n)(D-\gamma+1+\alpha)} P(x, d / d x)\right]^{m} x^{n} .
$$

It is interesting to note that if one extends the Heun equation by adding a term, $-\sigma / x$, the above analysis holds and yields polynomial solutions. Starting from

$$
\left[\frac{d^{2}}{d x^{2}}+\left(\frac{\gamma}{x}+\frac{\delta}{x-1}+\frac{\epsilon}{x-c}\right) \frac{d}{d x}+\frac{\alpha \beta x-q-\sigma / x}{x(x-1)(x-c)}\right] Y_{n}(x)=0,
$$


and performing a similarity transformation by writing $Y_{n}(x)=x^{1-\gamma+\sigma} \chi_{n}(x)$, and by imposing the constraint $q=(1-\gamma+\sigma)[(1+c) \sigma+\delta c+\epsilon]$ (instead of the usual one $\gamma+\delta+\epsilon=\alpha+\beta+1$ ), one finds that $\chi_{n}(x)$ obeys the following differential equation,

$$
\begin{array}{r}
{\left[D^{2}+(2 \sigma+1-\gamma+\delta+\epsilon) D+(1-\gamma+\sigma)(\sigma+\delta+\epsilon)+\alpha \beta\right.} \\
-(1+c) x \frac{d^{2}}{d x^{2}}-\{[2(1+\sigma)-\gamma](1+c)+\delta c+\epsilon\} \frac{d}{d x} \\
\left.+c \frac{d^{2}}{d x^{2}}+\frac{[2(1+\sigma)-\gamma] c}{x} \frac{d}{d x}\right] \chi_{n}(x)=0
\end{array}
$$

In the above case,

$$
F(D)=D^{2}+(2 \sigma+1-\gamma+\delta+\epsilon) D+(1-\gamma+\sigma)(\sigma+\delta+\epsilon)+\alpha \beta,
$$

and the rest of the terms belong to $P(x, d / d x)$. Imposing $F(D) x^{\lambda}=0$ yields,

$$
\lambda^{2}+(2 \sigma+1-\gamma+\delta+\epsilon) \lambda+(1-\gamma+\sigma)(\sigma+\delta+\epsilon)+\alpha \beta=0 \quad,
$$

which has two roots, $\lambda_{ \pm}$. In order to have polynomial solutions, either $\lambda_{+}$or $\lambda_{-}$or both must be a positive integer. The solutions can then be written as

$$
Y_{\lambda_{ \pm}}(x)=x^{1-\gamma+\sigma} \sum_{m=0}^{\infty}(-1)^{m}\left[\frac{1}{\left(D-\lambda_{+}\right)\left(D-\lambda_{-}\right)} P(x, d / d x)\right]^{m} x^{\lambda_{ \pm}} .
$$

In conclusion, we analyzed the Heun equation by making use of a recently developed method to solve linear differential equations of arbitrary order. Apart from the series solution, we have obtained polynomial solutions to the Heun equation. Further, we extended the Heun equation by adding a term, $-\sigma / x$, and showed that polynomial solutions also exist in this case. The solutions found here may throw new light on physical problems involving Heun equation.

[1] Ronveaux A (ed.), 1995 Heun's differential equation, Oxford University Press, Oxford

[2] Maier R S, The 192 Solutions of Heun equation, math.CA/0408317

[3] Christ N H and Lee T D, 1975 Phys. Rev. D 12, 1606

Jatkar D P, Kumar C N and Khare A, 1989 Phys. Lett. A 142, 200

Khare A and Mandal B P, 1998 Phys. Lett. A 239, 197

[4] Bhaduri R K, Khare A, Law J, Murthy M V N and Sen D, 1997 J. Phys. A: Math. Gen. 30, 2557

[5] Suzuki M, Takasugi E and Umetsu H, 1998 Prog. Theor. Phys. 100, 491

[6] Takemura K, 2003 Commun. Math. Phys. 235, 467; 2004 J. Nonlinear Math. Phys. 11, 21

[7] Dorey P, Suzuki J and Tateo R, 2004 J. Phys. A 37, 2047

[8] Gurappa N, Panigrahi P K, Shreecharan T and Sree Ranjani S, 2001 Frontiers of Fundamental Physics 4, Eds: Sidharth B G and Altaisky M V, Kluwer Academic Plenum Publishers, New York, 269

Gurappa N, Panigrahi P K and Shreecharan T, 2003 J. Comput. Appl. Math. 160, 103

Gurappa N and Panigrahi P K, 2003 Phys. Rev. B 67, 155323

[9] Gradshteyn I S and Ryzhik I M, 1965 Tables of Integrals, Series and Products, Academic Press Inc. 